Communications in Physics, Vol.21, No. 4 (2011), pp. 301-308

\title{
SUPERSOLIDS OF HARDCORE - BOSONS ON A TRIANGULAR LATTICE
}

\author{
PHAM THI THANH NGA \\ Water Resources University, 175 Tay Son, Hanoi \\ NGUYEN TOAN THANG \\ Institute of Physics, VAST, 10 Dao Tan, Hanoi
}

\begin{abstract}
We study the boson model on a triangular lattice interacting only via on-site hardcore repulsion by mapping to a system of spins $(S=1 / 2)$. We investigate the supersolid phase of the systems which is a state matter displaying both diagonal long-range (solid) order as well as off-diagonal long-range (superfluidity) by utilizing a semionic representation for the spin- $X X Z$ model. We show that the supersolid order is stable in the mean-field theory for a broad region of parameters. The inclusion of spin wave corrections modifies this picture, but the supersolid phase is still quite robust on the triangular lattices.
\end{abstract}

\section{INTRODUCTION}

Supersolidity where superfluid and crystalline orders coexist was firstly theoretically conjectured by Andreev and Lifshitz in 1969 [1] and then was developed by Leggett [2] and Chester [3]. A supersolids phase is a state of matter exhibiting simultaneously off diagonal (ODLRO) and diagonal long range order (DLRO). In $2004 \mathrm{Kim}$ and Chan [4,5] proved the existence of the supersolid state in solid $H e^{4}$ at temperatures below $\mathrm{T}=0.2$ $\mathrm{K}$ by measuring a tine superflow in a torsional oscillation experiment. This landmark experiment has attracted a lot of interest in the supersolid state and subsequently many new theories were proposed [6-11]. However, the true nature of the supersolids state is still unclear. One of the simplest models has been applied to study the possibility of the sufersolidity is the Quantum Gas Model (QGM) [12, 13]. Since the QGM can be exactly mapped to $\mathrm{s}=1 / 2$ quantum model [14] one can use helpful methods developed in quantum spin systems for understanding the physics underlying the sufersolidity. In particular, upon exploiting a mapping between hardcore latlice gas models and spin - 1/2 Heisenberg model, Liu and Fisher [13] have shown that within a mean - field approximation a supersolid phase exists in systems with a finite range of interactions between the bosons. Recent experiments in the realisation of optical lattices in ultracold atomic system motivate a search for a lattice supersolid, in particular in low dimensional and frustrated systems. In the mean time interest in hardcore bosons on two dimensional lattices has been on the rise (see [7] and Ref. therein). Recent studies in the two dimensional square lattice system revealed that quantum fluctuations play crucial role for the sufersolid phase. Namely they dramatically change the behavior and may even suppress the supersolid phase which is supposed to exist within the mean field theory [7]. A model of strongly interaction hardcore 
bosons on a triangular lattice is another open issue where the nature of the state for the hardcore bosons with frustrated nearest neighbor hoping has not been well understood $[15$ - 19].

In this report we study supersolidity in the triangular lattice using a semionic representations for spin operators, suggested by Popov and Fedotov [19]. Contrary to other methods which are based on the use of a Lagrange multiplier [20] leading to an average occupations the Popov - Fedotov procedure avoids this approximate treatment by introducing of an imaginary chemical potential. In the previous report we applied the Popov - Fedotov trick for a $1 / 2$ - spin antiferromagnetic Heisenberg system on the triangular lattice [21 - 22]. The obtained results will be exploited on this report for considering the supersolid phase.

This paper is organized as follows: In Sec. II we provide a Model Hamiltonian with mapping to the XXZ spin - 1/2 model. In Sec. III and IV we describe classical and spin - wave results. Conclusions are presented in Sec. V.

\section{MODEL HAMILTONIAN}

We begin with a simple model of hardcore bosons at half filling on the triangular lattice interacting via nearest-neighbor repulsive term

$$
H=-t \sum_{\langle i, j\rangle}\left(b_{i}^{+} b_{j}+b_{j}^{+} b_{i}\right)+V \sum_{\langle i, j\rangle}\left(n_{i}-\frac{1}{2}\right)\left(n_{j}-\frac{1}{2}\right),
$$

where $b_{i}\left(b_{i}^{+}\right)$annihilates (creates) a hardcore boson on site $\mathrm{i}$ and $n_{i}=b_{i}^{+} b_{i}$ are boson number operators. $\langle i, j\rangle$ refer to nearest neighbor links of the triangular lattice. The transfer integral t sets the energy scale and we will shall mainly consider the frustrated boson hopping at $t<0$.

It has been proposed that bosonic lattice models can be realized by loading ultracold bosonic atoms in regular lattice and the interaction between the bosons can be induced by using the dipolar interaction on condensate [23]. The nearest neighbor repulsion $V$ promotes the formation of "solid" order, where the boson occupations fall into regular patterns, at special densities commensurate with the lattice. The transfer integral $t$ favors mobile bosons and consequently a superfluid phase at $T=0$.

Since atoms cannot penetrate each other there exist only one atom at a time on a lattice site and consequently $b_{i}^{+}$and $b_{i}$ are the operators of a hardcore bosons which obey the Bose commutation relations on different lattice sites.

$$
\left[b_{i}^{+}, b_{j}^{+}\right]=\left[b_{i}, b_{j}\right]=\left[b_{i}, b_{j}^{+}\right]=0 \quad(i \neq j)
$$

In addition the hardcore constraint should be enforced on order to exclude the multiple occupation of atoms at each lattice point corresponding to the atomic core. We impose the anti - commutators on identical sites:

$$
\begin{aligned}
\left\{b_{i}^{+}, b_{i}^{+}\right\} & =\left\{b_{i}, b_{i}\right\}=0, \\
\left\{b_{i}, b_{i}^{+}\right\} & =1
\end{aligned}
$$

From (3) we get $n_{i}$ having a value either 0 or 1 . Due to the unusual statistics of hardcore bosons there does not exist a Wick's theorem for the operators $b_{i}, b_{i}^{+}$and 
the perturbative field theory is not applicable. Hence in the following we transform the model (1) to an equivalent anisotropic Heisenberg spin model in order to use advanced methods developed for quantum spin systems. It is easy to check that the spin $\mathrm{S}=1 / 2$ operator obey the same commutator relations (2) - (3) as hardcore bosons do. Therefore it is feasible to replace the hardcore boson operators by spin operators:

$$
\begin{aligned}
b_{i}^{+} & =\left(S_{i}^{x}+i S_{i}^{y}\right) \\
b_{i} & =\left(S_{i}^{x}-i S_{i}^{y}\right) \\
n_{i} & =\frac{1}{2}+S_{i}^{z}
\end{aligned}
$$

With this mapping the hardcore boson Hamiltonian becomes the XXZ spin - 1/2 Hamiltonian:

$$
H=J \sum_{\langle i, j\rangle}\left[\Delta\left(S_{i}^{x} S_{j}^{x}+S_{i}^{y} S_{j}^{y}\right)+S_{i}^{z} S_{j}^{z}\right]
$$

where $J=V>0$ is antiferromagnetic longitudinal exchange, $\Delta=-\frac{2 t}{V}$ is the anisotropy parameter.

In the spin language the superfluid order is equivalent to the in-plane ordering of the spins at a nonzero wave vector, while a charge density wave (solid) order implies long range correlations of the $z$-component of spins also at a nonzerowave vector. Therefore the supersolid phase corresponds to the spin having their $x y$-component aligned ferromagnetically (superfluid) with the $z$-component also ordered at a nonzero wave vector (solid). In the following we consider the XXZ antiferromagnetic Heisenberg model [5] in the case of an easy axis with $V>-2 t>0$.

\section{CLASSICAL GROUND STATE}

In this section we will be following closely the analysis of Sheng and Henley [24]. The classical limit is obtained by setting $S=\infty$. In the ground state the triangular lattice breaks up into three sublattices $A, B, C$, within each sublattice the spins are ordered ferromagnetically and can be described by two polar angles $\left(\phi_{i}, \theta_{i}\right)(i=A, B, C$ sublattice). In the mean field solution the sublattice magnetization are co - planar, with the plane containing the easy axis. Let us choose it as the $z x$ - plane, i.e, $\phi_{A}=\phi_{B}=\phi_{C}=0$. By solving the equations:

$$
\frac{\partial H\left(\theta_{A}, \theta_{B}, \theta_{C}\right)}{\partial \theta_{i}}=0
$$

we get the following equations for classical value of $\theta_{i}$ :

$$
\begin{aligned}
& \Delta \cos \theta_{A}\left(\sin \theta_{B}+\sin \theta_{C}\right)=\sin \theta_{A}\left(\cos \theta_{B}+\cos \theta_{C}\right), \\
& \Delta \cos \theta_{B}\left(\sin \theta_{C}+\sin \theta_{A}\right)=\sin \theta_{B}\left(\cos \theta_{C}+\cos \theta_{A}\right), \\
& \Delta \cos \theta_{C}\left(\sin \theta_{A}+\sin \theta_{B}\right)=\sin \theta_{C}\left(\cos \theta_{A}+\cos \theta_{B}\right) .
\end{aligned}
$$

The three equations in (7) are not independent of each other [24]. Thus we are free to choose $\theta_{A}$ and parameterize the solutions of (7) as follows:

$$
\theta_{B}=\arctan \left(\frac{\tan \theta_{A}}{\Delta}\right)+\arccos \left(\frac{\Delta}{(1+\Delta)\left[\sin ^{2} \theta_{A}+\Delta \cos ^{2} \theta_{A}\right]^{1 / 2}}\right),
$$




$$
\theta_{C}=\arctan \left(\frac{\tan \theta_{A}}{\Delta}\right)-\arccos \left(\frac{\Delta}{(1+\Delta)\left[\sin ^{2} \theta_{A}+\Delta \cos ^{2} \theta_{A}\right]^{1 / 2}}\right) .
$$

where $\theta_{A} \in\left[0, \theta_{o}\right]\left(\sin \theta_{o}=\frac{\Delta}{1+\Delta}\right)$. From Eqs. (7) we can see that $\alpha_{1}=\left\{\theta_{1}, \theta_{2}, \theta_{3}\right\}$ is a set of solution, then:

$$
\begin{gathered}
\alpha_{2}=\left\{\theta_{2}, \theta_{3}, \theta_{1}\right\}, \\
\alpha_{3}=\left\{\theta_{3}, \theta_{1}, \theta_{2}\right\}, \\
\alpha_{4}=\left\{\pi-\theta_{1}, \pi-\theta_{2}, \pi-\theta_{3}\right\}, \\
\alpha_{5}=\left\{\pi-\theta_{2}, \pi-\theta_{3}, \pi-\theta_{1}\right\}, \\
\alpha_{6}=\left\{\pi-\theta_{3}, \pi-\theta_{1}, \pi-\theta_{2}\right\} .
\end{gathered}
$$

are equivalent sets of the solutions. So the classical ground state has the six - fold symmetry parameterized by the continuous parameter $\theta_{A}$.

The ground state energy is:

$$
\frac{E_{\text {colf }}}{N J S^{2}}=-\frac{1+\Delta+\Delta^{2}}{1+\Delta}
$$

The longitudinal magnetization $m_{z}$ is defined as:

$$
m_{z}=S\left(\cos \theta_{A}+\cos \theta_{B}+\cos \theta_{C}\right)
$$

and the transverse component is given by:

$$
m_{C}=S\left(\sin \theta_{A}+\sin \theta_{B}+\sin \theta_{C}\right)
$$

The ground state turns out to have the same absolute value of total magnetization:

$$
|m|=\frac{1-\Delta}{1+\Delta} S
$$

while $m_{z}=0$ Notice that (13) and (14) mean that the supersolid phase appears in a all region of the anisotropy parameter $0 \leq \Delta<1$.

\section{SPIN WAVE THEORY}

The ground state has a non - trivial continuous degeneracy parameterized by $\theta_{A}$ (non - trivial in the sense that the Hamiltonian of the system does not have the same symmetries as the spin configurations), so one expects the degeneracy to be lifted by quantum fluctuations. Here we present the spin-wave analysis by using Popov - Fedotov trick. The main complication is that all of the classical ground states parameterized by $\theta_{A}$ can be chosen as the starting point of a spin-wave calculation. We implement spin wave theory in the usual way. First we perform rotation in the local $z$-axis to the direction of the classical sublattice magnetization:

$$
\begin{gathered}
S_{i}^{x}=S_{i}^{x^{\prime}} \cos \theta_{i}+S_{i}^{z^{\prime}} \sin \theta_{i} \\
S_{i}^{z}=S_{i}^{z^{\prime}} \cos \theta_{i}+S_{i}^{x^{\prime}} \sin \theta_{i} \\
S_{i}^{y}=S_{i}^{y^{\prime}}
\end{gathered}
$$


for $i=A, B, C$. This leads to:

$$
H=-\frac{1}{2} \sum_{\langle i, j\rangle, \alpha, \beta} J_{i j}^{\alpha \beta} S_{i}^{\alpha} S_{j}^{\beta}
$$

where:

$$
\begin{aligned}
J_{i j}^{x x} & =-2 J\left(\cos \theta_{i} \cos \theta_{j}+\Delta \sin \theta_{i} \sin \theta_{j}\right) \\
J_{i j}^{y y} & =-2 J \\
J_{i j}^{z z} & =-2 J\left(\sin \theta_{i} \sin \theta_{j}+\Delta \cos \theta_{i} \cos \theta_{j}\right) \\
J_{i j}^{x z} & =J_{i j}^{z x}=-2 J\left(\cos \theta_{i} \cos \theta_{j}-\Delta \sin \theta_{i} \sin \theta_{j}\right) \\
J_{i j}^{x y} & =J_{i j}^{y x}=J_{i j}^{z y}=J_{i j}^{y z}=0
\end{aligned}
$$

In the Popov - Fedotov formalism the partition function of the Hamilltonian (15) is given by the coherent state functional integral:

$$
Z=\frac{1}{i^{N}} \int D\left[a_{i \sigma}^{*}(\tau) a_{i \sigma}(\tau)\right] \exp (-S)
$$

where

$$
S=\int_{\alpha}^{\beta} d \tau\left[\sum_{i \sigma} a_{i \sigma}^{*}(\tau) \partial_{\tau} a_{i \sigma}(\tau)+H\left(a_{i \sigma}^{*}(\tau) a_{i \sigma}(\tau)\right)+\frac{i \pi}{2 \beta} \sum_{i, \sigma=1,2} a_{i \sigma}^{*}(\tau) a_{i \sigma}(\tau)\right]
$$

$a_{i \sigma}^{*}$ and $a_{i \sigma}(\tau)$ are the Grassmann variables corresponding to the pseudo fermi operators $a_{i \sigma}^{*}$ and $a_{i \sigma}(\tau)$. The spin vectors are given as:

$$
\vec{S}_{\tau}=\frac{1}{2} a_{i \sigma}^{*} \vec{\tau}_{\sigma \sigma^{\prime}} a_{i \sigma^{\prime}}
$$

where $\vec{\tau}$ are the Pauli matrices. The last form in (18) was introduced to eliminate the contribution of the unphysical states from the partition function [19]. Following the same way as in [22] we get the partition function (17) in term of the auxiliary Bose fields $\vec{\varphi}_{i}$ :

$$
Z=\frac{1}{Z_{o}} \int D \vec{\varphi} e^{-S_{e f f}[\varphi]}
$$

The effective action $S_{\text {eff }}$ is given by:

$$
S_{e f f}[\varphi]=\int_{0}^{\beta} d \tau S_{0}[\vec{\varphi}]-\ln \operatorname{det} \beta K_{i}
$$

with auxiliary field action $S_{0}$ :

$$
S_{0}[\varphi(\tau)]=\frac{1}{2} \sum_{<i j>\alpha, \beta}\left(J^{-1}\right)^{\alpha \beta} \vec{\varphi}_{i}(\tau) \vec{\varphi}_{j}(\tau)
$$

and $\left(J^{-1}\right)_{i j}^{\alpha \beta}$ is the inverse of the coupling matrix $J_{i j}^{\alpha \beta} . Z_{0}$ is auxiliary partition function:

$$
Z_{0}=\int D \vec{\varphi} e^{-i \int_{0}^{\beta} d \tau S_{0}[\varphi(\tau)]}
$$


The matrix $K_{i}$ in the frequency representation reads:

$$
\widehat{K}_{i}\left(\omega_{1}, \omega_{2}\right)=\left(-i \omega_{1}-\frac{i \pi}{2 \beta}\right) \delta_{\omega_{1}, \omega_{2}} \widehat{I}+\frac{1}{2} \vec{\sigma} \vec{\varphi}_{i}\left(\omega_{1}-\omega_{2}\right)
$$

Decomposing the matrix $\widehat{K}$ into nonperturbation and perturbation parts:

$$
\widehat{K}=\widehat{K_{0}}+\widehat{M}
$$

And expanding $\operatorname{Tr}(\ln \widehat{K})$ in a Taylor series:

$$
\operatorname{Tr}(\ln \widehat{K})=\operatorname{Tr}\left(\ln \widehat{K_{0}}\right)+\operatorname{Tr} \sum_{n=1}^{\infty} \frac{(-1)^{n+1}}{n}\left(K_{0}^{-1} \widehat{M}\right)^{n}
$$

It's straightforward to get the mean field free energy:

$$
F_{m f}=\frac{1}{\beta} \sum_{i j, \alpha \beta}\left(J^{-1}\right)_{i j}^{\alpha \beta} \varphi_{i 0}^{\alpha} \varphi_{j 0}^{\beta}+\frac{1}{\beta} \sum_{i} \ln 2 \cosh \frac{\beta\left|\varphi_{i 0}\right|}{2}
$$

where $\varphi_{i 0}^{\alpha}$ is the mean field Hubbard - Stratonovich auxiliary field:

$$
\vec{\varphi}_{i}(\Omega)=\vec{\varphi}_{i 0}(\Omega=0)+\delta \vec{\varphi}(\Omega)
$$

Utilizing the transformation in $\mathrm{Eq}(14)$ we can set $\vec{\varphi}_{00}=\left(0,0, \varphi_{0}\right)$. The first order fluctuation $\delta \vec{\varphi}$ gives no contribution. To the second order in the fluctuations the effective action reads:

$$
S_{e f f}^{(2)}=\sum_{i j \Omega} D_{i j}^{\alpha \beta}(\Omega) \delta \varphi_{i}^{\alpha}(\Omega) \delta \varphi_{j}^{\beta}(-\Omega)
$$

where

$$
\begin{gathered}
D_{i j}^{\alpha \beta}(\Omega)=\frac{\beta}{2}\left[\left(J^{-1}\right)_{i j}^{\alpha \beta}+M_{i}^{\alpha \beta}(\Omega) \delta_{i j}\right] \\
M_{i}^{x x}(\Omega)=M_{i}^{y y}(\Omega)=-i M_{i}^{x y}(\Omega)=i M_{i}^{y x}(\Omega)-\frac{1}{2} \frac{\tanh \varphi_{0}}{\left(\varphi_{0}-i \Omega\right)}
\end{gathered}
$$

with

$$
\begin{gathered}
M_{i}^{z z}(\Omega)=-\frac{1}{4} \frac{\delta_{\Omega, 0}}{\cosh ^{2} \frac{\beta \varphi_{0}}{2}} \\
M_{i}^{y z}(\Omega)=M_{i}^{z y}(\Omega)=M_{i}^{x z}(\Omega)=M_{i}^{z x}(\Omega)=0
\end{gathered}
$$

Because of the three sublattice structure, translational invariance is valid only within sublattices, and the Bloch vector $\vec{k}$ have to be chosen from the correspondingly reduced magnetic Brillouin zone. It is therefore helpful to introduce three kind of the fluctuation $\delta \varphi_{A}, \delta \varphi_{B}, \delta \varphi_{C}$; the corresponding k-space representations are $\delta a(k), \delta b(k), \delta c(k)$. Performing the Fourier trarnsformation, the effective action can be written in the compact matrix form.

$$
S^{e f f}[\delta \Phi]=\sum_{k, \alpha, \beta, \Omega} \overline{\delta \Phi}^{\alpha}(k, \Omega) D^{\alpha \beta}(k, \Omega) \delta \Phi^{\alpha}(k, \Omega)
$$

where $D^{\alpha \beta}(k, \Omega)$ is Fourier trarnsformation of $D_{i j}^{\alpha \beta}(k, \Omega)$ and the vector $\delta \Phi^{\alpha}(k, \Omega)$ is introduced as follows:

$$
\delta \Phi^{\alpha}(k, \Omega)=\left(\delta a_{k}^{\alpha}(\Omega), \delta b_{k}^{\alpha}(\Omega), \delta c_{k}^{\alpha}(\Omega)\right)
$$


The magnon energy is the solution of the following equation:

$$
\operatorname{det}\left\|D^{\alpha \beta}(k, \Omega)\right\|=0
$$

The $D^{\alpha \beta}(k, \Omega)$ is $9 \times 9$ matrix, but the elements containing $z$-component are decoupled, so only the $6 \times 6$ matrix remains. Since carrying though the calculation analytically is not possible for general $\Delta$, we omit further formal details and go over to discuss limiting cases and numerical results.

For $\Delta=0$ we regain the results for isotropic Heisenberg model, where the magnon spectrum is given by:

$$
\omega(k)=3 J S \sqrt{(1-\gamma(k))(1+2 \gamma(k))}
$$

with

$$
\gamma(k)=\frac{1}{3}\left(\cos k_{x}+2 \cos \frac{k_{x}}{2} \cos \frac{\sqrt{3}}{2} k_{y}\right)
$$

For general $\Delta<1$, in the small $k$ limit we have:

$$
\begin{aligned}
\omega_{1}(k) \omega_{2}(k) \omega_{3}(k)= & \frac{9 \Delta^{2} J^{3}}{\sqrt{61}}\left(1+\frac{1}{1+\Delta}\right)^{2}\left[\left(k \delta_{1}\right)^{2}+\left(k \delta_{2}\right)^{2}+\left(k \delta_{3}\right)^{2}\right] \times \\
& \times \sqrt{(2 \Delta+1)(1-\Delta)}
\end{aligned}
$$

The ground state energy turns out to be lowest when $\theta_{\Delta}=0$ and decreases with $\Delta$ increasing. For small anisotropy parameter $\Delta<<1$ the ground state energy with the spin wave correction is:

$$
E_{s \omega}=-N S(S+1) J \frac{1+\Delta+\Delta^{2}}{1+\Delta}-S \Delta J
$$

For the $\theta_{\Delta}=0$ ground state, the sublattice magnetizations are reduced due to spin wave corrections. This spin reductions depend on the anisotropy parameter $\Delta<1$. However, the total $z$-component of magnetization doer not vanish until $\Delta=0$, which means the Neel solid order exists for $0 \leq \Delta<1$.

\section{CONCLUSIONS}

We have shown that supersolid order is stable in mean - field theory for abroad region of anisotropy parameter $\Delta$. The spin wave corrections select the true ground state from continuously degenerate classical ground state manifold. However the supersolid is quite robust on the triangular lattice. The underlying reason is that the frustration prevents all the particles from condensing into a "solid". Our result is complimentary to those of [15], where one considers the unfrustrated hopping case. We also reproduce some results of the numerical study [16 - 18]. It is not surprise because spin wave theory seems sufficient for the triangular lattice. We close with a open important question concerning the effects of nonzero temperature on the system. Of course, since this is a two - dimensional system, the Bose condensate disappears for $T \neq 0$, but power - law ODLRO is expected to remain. The quantum thermal fluctuation and frustration may compete to produce various phases as the temperature is raised. It would be interesting to investigate this case by Popov Fedotov approach. 


\section{ACKNOWLEDGMENT}

This research is supported by NAFOSTED (103.027.809).

\section{REFERENCES}

[1] A. Andreev and I. Lifshits, Sov. Phys. JETP., 29 (1969) 1769.

[2] A. J. Leggett, Phys. Rev. Lett. 25 (1970) 1543.

[3] G. V. Chester, Phys. Rev. A2 (1970) 256.

[4] E. Kim and M. H. W. Chan, Nature 427 (2005) 225.

[5] E. Kim and M. H. W. Chan, Science 305 (2004) 194.

[6] A. J. Stoffell and M. Gulacsi, Eur. Phys. J. B67 (2009) 169.

[7] K. Ng, Y. C. Chen and Y. C. Tzeng, J. Phys. Cond. Matt. 22 (2010) 185600.

[8] P. Chen, C. Lai and M. Yang, Phys. Rev. B81 (2010) 020409 (R).

[9] C. D. Yoo and A. T. Porsey, Phys. Rev. B81 (2010) 134518.

[10] L. Seabra and N. Shannon, Phys. Rev. Lett. 104 (2010) 237205.

[11] H. T. Veda and K. Totsuka, Phys. Rev. B81 (2010) 054442.

[12] H. Matsubara and T.Tsuneto, Prog. Theor. Phys. 46 (1970) 411.

[13] K-S. Lin and M.F. Fisher, J. Low. Temp. Phys. 10 (1973) 655.

[14] T. Matsubara and H. Matsuda, Prog. Theor. Phys. 17 (1957) 19.

[15] G. Murthy, P. Arovar and A. Auerbach, Phys. Rev. 55 (1997) 3104.

[16] F. Wang, F. Pollmann and A. Vishwanath, Phys. Rev. Lett. 102 (2009) 017203.

[17] H. C. Jiang et al., Phys. Rev. B 79 (2009) 020409.

[18] D. Heidarian and A. Paramekanti, Phys. Rev. Lett. 104 (2010) 015301.

[19] V. N. Popov and S. A. Fedotov, Sov. Phys. JETP 67 (1988) 535.

[20] A. Auebach, Interacting electrons and quantum magnetism, Springer. Verlag (1994).

[21] Tran Van Quang, Pham Thi Thanh Nga and Nguyen Toan Thang, Comm. Phys. 19 (2009) 71.

[22] Pham Thi Thanh Nga and Nguyen Toan Thang, Comm. Phys. 20 (2010) 295.

[23] K. Goral et al., Phys. Rev. Lett. 88 (2002) 170406.

[24] Q. Sheng and C. L. Henley, J. Phys. Condens. Matter. 4 (1992) 2937.

Received 10 October 2010. 